\title{
Entre adverbes, noms et pronoms : le cas des modifieurs temporels
}

\author{
Sylvain Kahane \\ Modyco, Université Paris 10 \& CNRS / Alpage, INRIA \\ sylvain@kahane.fr \\ www.kahane.fr
}

«Ce que nous allons chercher à des milliers de kilomètres ou tout près, ce sont des moyens supplémentaires de comprendre comment fonctionne l'esprit humain. » (Lévi-Strauss, 1988)

\section{Introduction}

Dans cet article, nous nous intéresserons aux modifieurs temporels généralement classés parmi les adverbes, modifieurs qui expriment un moment (Il venait autrefois), une durée (Il venait longtemps) ou encore une fréquence (Il venait toujours). Outre leur origine nominale évidente (autre fois, long temps, tous jours), ces adverbes commutent avec des syntagmes nominaux. Nous allons donc discuter de la relation qu'entretient cette classe d'adverbes avec celle des noms et plus encore avec celle des pronoms. Ceci nous amènera à redéfinir les liens entre ces trois parties du discours ${ }^{1}$ et à introduire la catégorie des substantifs englobant pronoms et noms propres.

Cet article n'introduit pas de données réellement nouvelles. Notre objectif est plutôt de questionner les choix traditionnellement faits dans la présentation des parties du discours, notamment en linguistique française. Les modifieurs temporels sont l'occasion 1) de montrer à nouveau (voir Comrie 1981 : 109 ou Schachter 1985) que les parties du discours ne forment pas nécessairement une partition des lexèmes dans le sens où un lexème peut posséder des propriétés caractéristiques de différentes parties du discours et 2) de défendre l'importance de considérer une partie du discours distincte des noms, les substantifs, dont nous pensons qu'elle est entre autres nécessaire pour comprendre les relations qu'il peut y avoir entre noms, pronoms et adverbes. Insistons tout de suite sur le fait que nous utilisons le terme substantif dans une acception particulière, que nous aurons l'occasion de situer précisément par rapport aux précédents emplois de ce terme.

Notre étude comportera trois sections. Dans la section 2, nous étudierons le syntagme nominal et les définitions du nom et du pronom et nous introduirons la notion de substantif considérée ici. Dans la section 3, nous présenterons une définition distributionnelle des parties du discours à la Tesnière qui nous permettra à la fois de préciser ce qui caractérise les parties du discours du français dans la diversité des langues et comment se situent adverbes et noms dans cette caractérisation générale des parties du discours. La section 4 sera consacrée au cas des syntagmes nominaux modifiant un verbe, ce qui nous amènera à discuter les relations entre noms, substantifs et adverbes.

\section{Substantif, nom, pronom et déterminant}

\subsection{Substantif et nom}

Les termes nom et substantif ont une origine commune dans la description grammaticale scholastique qui parle de nomen substantivum. Il s'agit d'une unification de deux notions essentielles de la philosophie

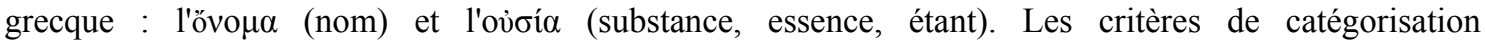
essentiellement morphologiques et les similitudes flexionnelles en grec ainsi qu'en latin ont pour conséquence qu'on subsume sous le terme övo $\mu \alpha /$ nomen ce qu'on appelle aujourd'hui des noms et des adjectifs (Almeida \& Maillard 1998). Etant donné que l'on nomme aujourd'hui nom ce que l'on nommait 
avant (nom) substantif, nous considérons que le terme substantif est à nouveau libre ${ }^{2}$ et nous souhaitons le réinvestir dans un sens légèrement différent et finalement plus conforme à son étymologie. Nous appellerons catégorie du substantif la classe des unités syntaxiques pouvant occuper les positions de sujet et d'objet direct. En français, les substantifs se rencontrent également derrière les prépositions. On désignera plus précisément par substantif les mots et les lexèmes qui peuvent former à eux seuls un substantif, comme les pronoms personnels et les noms propres. Lorsqu'il s'agit de syntagmes plus complexes, nous parlerons de groupes substantivaux.

Cet emploi du terme substantif peut être imputé à Lemaréchal (1985). Il nous semble bien approprié, car les substantifs réfèrent généralement à une entité possédant une substance, c'est-à-dire un objet du monde observable et palpable. Il faut noter tout de suite que cette catégorie ne comprend pas les noms communs. En français, un nom comme chat n'a pas la propriété de désigner seul un objet du monde, ni d'être le sujet ou l'objet direct d'un verbe. Le nom chat dénote plutôt une classe d'objets du monde ( "les chats ») ou une propriété (« être un chat »), mais la référence à un objet du monde, c'est-à-dire à un chat particulier, nécessite la combinaison du nom avec un déterminant : un chat, le chat, ce chat, etc. De même, lorsque nous disons que les noms propres sont des substantifs, nous parlons de noms propres tels que François, Chirac ou Paris, mais pas de noms comme France ou Français qui nécessitent un déterminant ${ }^{3}$.

Si les noms doivent être combinés à un déterminant pour former un groupe substantival, ils peuvent néanmoins être utilisés sans déterminant. Les emplois du nom sans déterminant confirment le fait que le nom nu en français ne possède pas la propriété de référence :

(1) a. Pierre a trouvé un manche de pioche dans le grenier.

b. Pierre est docteur. Il n'a pas besoin de nous.

c. Nous avons parlé voitures.

En (1a), pioche ne réfère pas et la reprise pronominale est impossible :

(1) d. \#Pierre a trouvé un manche de pioche dans le grenier. Elle appartenait à son père.

En (1b), il s'agit d'emplois prédicatifs du nom : le nom est intégré à la construction verbale. L'emploi en (1c) est plus étrange, même s'il est clair qu'ici le complément renvoie davantage à la classe entière des voitures qu'à des voitures en particulier. Ils existent néanmoins des constructions où un nom sans déterminant forme un groupe substantival référentiel :

(1) e. Parents et enfants seront accueillis dans le réfectoire.

Notons que le fait que le nom tende à ne pas être accompagné de ses marqueurs habituels (la définitude dans le cas du français) lorsqu'il ne forme pas un groupe référentiel est une propriété assez générale des langues (Hopper \& Thompson 1984). On peut considérer malgré tout, comme il est d'usage, que le lexème nominal possède la propriété de référer et qu'il s'agit même là d'une propriété définitoire de la classe des noms. Sans nécessairement remettre en question ce point de vue, nous souhaitons insister sur le fait que l'actualisation de la référence passe par la combinaison du nom avec certains marqueurs et que ceci distingue les noms des substantifs qui actualisent la référence sans l'ajout d'aucun marqueur.

\subsection{Substantif et pronom}

Une fois cette distinction établie entre nom et substantif, intéressons-nous au pronom. Bien que figurant parmi les parties du discours traditionnelles, la classes des pronoms possède un statut bien particulier : les pronoms appartiennent à une classe qui est transversale et coupe à travers les autres parties du discours. Une partie importantes des pronoms appartiennent à la classe des substantifs ${ }^{4}$ : nous les appellerons les pronoms substantivaux. Il n'y a par contre quasiment aucun pronom qui appartienne à la classe des noms. Le seul exemple en français est la série mien, tien, ..., leur, qui en plus forme une séquence figée avec l'article défini, séquence qui est, elle, un substantif ${ }^{5}$. Il existent d'autres pronoms non substantivaux comme les pronoms relatifs qui ont un rôle de complémenteur (en plus de leur rôle substantival) ou les 
pronoms possessifs (mon livre) qui fonctionnent comme déterminant. D'autres langues possèdent des verbes pronominaux comme (to) do en anglais (voir également Schachter 1985 pour d'autres exemples) ${ }^{6}$. Il est assez difficile de donner une définition générale des pronoms. La première caractéristique est plutôt sémantique : un pronom est un élément qui sert à désigner un élément du contexte et dont le référent est entièrement déterminé par ce contexte par une relation déictique ou anaphorique ${ }^{7}$. Autrement dit, il n'y a pas une classe d'objet du monde que l'on peut dénoter par moi, elle, on, ça ou celui-là ; ces éléments vont déclencher un processus d'instanciation dans un énoncé donné dans une situation donnée. A cette caractéristique sémantique s'ajoutent certaines caractéristiques syntaxiques qui tendent à faire des pronoms des éléments plus grammaticaux que les autres lexèmes. Par exemple, en français, les pronoms personnels sont les seuls lexèmes à varier en cas et à alterner des formes fortes avec des formes faibles qui se cliticisent sur le verbe. Ils se distinguent ainsi par des propriétés morphologiques ou d'ordre des mots.

La distinction entre pronoms (substantivaux) et noms propres mérite d'être discutée. Nous les regroupons dans une même catégorie syntaxique, la catégorie du substantif, en raison de leur distribution similaire, puisqu'ils peuvent être utilisés seuls comme sujet ou objet direct :

(2) a. Paris me plaît. J'aime Paris.

b. Ça me plait. J'aime ça.

Ils possèdent pourtant une propriété sémantique antinomique : alors que les pronoms instancient un référent en fonction de leur utilisation dans un contexte particulier, les noms propres, comme Paris ou Chirac, possèdent une référence absolue. En fait, il y a une sorte de continuum sémantique entre les pronoms et les noms propres à référence absolue. Un lexème comme maman, lorsqu'il est utilisé comme substantif (c'est-à-dire sans déterminant) possède une propriété déictique : il ne réfère pas nécessairement à une élément du contexte immédiat, mais son référent doit être calculé en fonction du contexte d'énonciation. Dans Demande à maman !, maman ne peut désigner que la mère du locuteur ou de son (jeune) interlocuteur. Nous dirons qu'il s'agit d'un déictique indirect par opposition à un déictique direct comme moi, qui appartient nécessairement au contexte d'énonciation et peut être ainsi désigné. Les prénoms n'ont pas non plus de référent absolu. Dans Demande à Anne !, l'interprétation de Anne dépend des personnes prénommées Anne que connaissent en commun les interlocuteurs et qui sont «actives » dans le contexte d'énonciation. Et plus les locuteurs connaissent d'Anne et plus on se rapproche du fonctionnement d'un pronom anaphorique comme lui dans Demande-lui !

Il y aussi beaucoup d'éléments qui sont clairement des substantifs, mais qu'il est difficile de caractériser sur des critères sémantiques comme des pronoms, puisqu'ils commutent avec des syntagmes nominaux de sens équivalents. C'est par exemple le cas des formes substantivées du nom chose : quelque chose ou grand-chose.

(3) a. Je t'ai demandé une chose (importante).

b. Je t'ai demandé quelque chose (d'important).

(4) a. Il ne fait pas beaucoup de choses (intéressantes).

b. Il ne fait pas grand-chose (d'intéressant).

Si leur statut pronominal est loin d'être clair (bien qu'ils soient traditionnellement appelés des pronoms indéfinis), il est clair qu'il s'agit de substantifs et non de noms, puisqu'ils ne prennent pas de déterminants (ou en intègre un comme quelque chose). Il s'agit de plus d'une sous-classe de substantifs qui se caractérise par le fait que les adjectifs qui les modifient doivent être introduits par la préposition de (cf. (3b) et (4b)), ce qui les éloigne encore de la classe des noms.

\subsection{Substantif et déterminant}

Comme les noms ne peuvent former à eux seuls un groupe substantival, la question a été souvent posée de savoir qui du nom ou du déterminant apporte la « fonction substantivale », c'est-à-dire lequel des deux 
éléments contrôle la distribution du groupe qu'ils forment et lui permet d'occuper une position où un substantif est attendu. La réponse n'a jamais été tranchée de façon définitive, même si la plupart des théories syntaxiques, notamment celles qui font l'hypothèse qu'un constituant syntaxique possède une tête syntaxique, sont amenées à trancher. Il en est ainsi de la grammaire générative qui, depuis Abney (1987), a adoptée la «DP-hypothesis » et considère que le groupe substantival est un Determiner Phrase, après avoir longtemps considéré qu'il s'agissait d'un Noun Phrase. Nous n'allons pas vraiment nous pencher sur cette question, d'autant que nous pensons qu'elle ne possède pas de réponse binaire et que c'est la notion même de tête syntaxique qu'il faudrait discuter. Dans cette section, nous allons étudier les liens réels qui existent entre substantifs et déterminants.

Comme on l'a vu, le nom nu en français ne possède pas la propriété de référence. Celle-ci est amenée par le déterminant. En particulier, l'opposition entre articles défini et indéfini - le chat vs un chat - indique si le référent du groupe substantival est déjà connu ou non des interlocuteurs. Cette propriété de référence caractérise également les pronoms et il y a en français une certaine perméabilité entre déterminants et pronoms :

\section{(5) Une photo gisait sur la moquette. Plusieurs (autres) étaient étalées sur le bureau.}

Dans cet emploi, plusieurs est souvent appelé un pronom indéfini. Il s'agit ici d'une anaphore nominale et plusieurs est bien un pro-nom (plusieurs = plusieurs photos) et non un pro-substantif (puisque plusieurs ne renvoie pas un référent préexistant dans le contexte) comme le sont généralement les éléments appelés traditionnellement pronoms. Dans la suite, nous distinguerons entre pro-nom, déclenchant une anaphore nominale, et pro-substantif, déclenchant une coréférence ou la référence à un élément du contexte. L'article défini donne un bon exemple de la perméabilité entre les trois classes - déterminant, pro-nom et pro-substantif. En français, l'article défini peut être combiné avec un nom ou avec un adjectif, mais le fonctionnement de l'article diffère dans les deux cas. Ceci est bien illustré par la paire minimale suivante proposée par Creissels (1995) :

(6) a. Je n'aime pas la jupe rouge. Je vais prendre la robe.

\section{b. Je n'aime pas la jupe rouge. Je vais prendre la bleue.}

c. Je n'aime pas la jupe rouge. Je ne vais pas la prendre.

Dans le premier cas, la robe n'est pas rouge a priori, alors que dans le deuxième cas, la bleue est nécessairement une jupe, c'est-à-dire que l'article y fonctionne comme un pro-nom. On peut alors être tenté de considérer que dans le troisième cas, la forme la est encore une forme du même élément qui fonctionne cette fois-ci comme pro-substantif. On peut toutefois en être dissuadé par le fait que si d'autres déterminants fonctionnent comme pro-nom, presqu'aucun ne fonctionne comme pro-substantif ${ }^{8}$. Au delà des liens entre substantifs et déterminants, ces exemples mettent en évidence différentes stratégies possibles dans la définition des catégories syntaxiques. Ou bien l'on considère que les différents emplois d'un lexème (par exemple plusieurs comme déterminant et pro-nom ou la comme déterminant, pro-nom et pro-substantif) définissent une catégorie qui se caractérise justement par cette diversité d'emplois, ou bien l'on veut au contraire restreindre le nombre de catégories et considérer que de tels éléments sont polycatégoriels. Dans ce dernier cas, la notion de catégorie est plus nettement liée à celle de fonction, une fonction particulière impliquant une catégorie particulière. Nous allons discuter ce point maintenant ${ }^{9}$.

\section{Catégories et fonctions}

Nous allons répondre à la question précédente en précisant notre cadre théorique. Nous nous plaçons dans un cadre dans lequel nous distinguons clairement catégories et fonctions. Une fonction est liée à une position structurale : elle est définie par un paradigme positionnel, c'est-à-dire par l'ensemble des syntagmes qui peuvent occuper cette position. La catégorie, elle, est définie par l'ensemble des positions que peut occuper un syntagme. Il peut y avoir des catégories différentes qui occupent la même position ( $\mathrm{il}$ pèse lourd/une tonne) et des fonctions différentes occupées par la même catégorie (il est drôle, un garsdrôle, un drôle de gars). Mais il y a aussi une certaine convergence entre catégorie et fonction. En 
particulier, certaines positions tendent à être occupées par une catégorie spécifique de syntagmes. C'est par exemple le cas de la position déterminative du groupe substantival et on appelle généralement déterminant aussi bien les éléments qui occupent la position déterminative quelle que soit leur catégorie que les éléments dont la catégorie est caractérisée pas l'occupation de cette position.

Notre définition des catégories et des fonctions suppose une analyse structurale préalable. Pour définir les catégories, il faut d'abord identifier les positions syntaxiques. Pour cela, nous utiliserons une méthodologie développée par Jespersen (1924) et Tesnière (1959) et souvent reprise par les travaux en typologie des langues. Cette méthodologie repose sur l'hypothèse que la phrase possède une structure essentiellement hiérarchique et que les catégories peuvent être définies de manière récursive en partant des éléments prédicatifs. Notre approche des parties du discours en français présente donc deux caractéristiques : 1) sans rejeter la classification traditionnelle, nous souhaitons nous en départir en étudiant le français selon la méthodologie généralement employée pour les langues exotiques nouvelles ; 2) non seulement nous nous basons sur l'analyse distributionnelle, mais sur une analyse distributionnelle qui s'appuie sur une analyse structurale préalable.

Le fait d'appuyer notre analyse distributionnelle sur une analyse structurale préalable ne va pas de soi. On a souvent l'impression que les constructions de modèles procèdent à l'inverse. Dans la mesure où les approches formelles justifient réellement leurs choix et explicitent leur démarche, il semble que beaucoup d'approches, notamment celles issues de la syntaxe X-barre, effectuent d'abord une catégorisation des constituants avant d'étudier les relations structurelles, ces relations n'étant en fait même pas envisageables sans une catégorisation préalable. On ne voit par exemple jamais de travaux qui commencent par définir des structures syntagmatiques non étiquetées avant d'introduire les étiquettes catégorielles. Comme le note Croft $(2005: 66)$ à propos des présentations de la grammaire générative, et notamment d'Haegeman (1994), les parties du discours ne sont pas discutées et sont supposées universelles sans qu'on donne aucune propriété permettant de les caractériser dans une langue donnée. Ceci amène à projeter sans précaution les catégories des langues déjà étudiées sans en tester réellement la pertinence, comme l'a fait la grammaire traditionnelle des langues modernes européennes avec les catégories du grec ou du latin (ce qui, étant donné leur forte parenté, était encore relativement raisonnable).

Nous considérons qu'il n'y a pas de définition universelle des parties du discours et que les grandes classes distributionnelles sont propres à chaque langue et caractérisées par des critères internes à la langue (sur la même position cf. par exemple Schachter 1985 ou Hengeveld 2004). Par contre, nous pensons qu'il existe une stratégie universelle pour définir les parties du discours, qui consiste à partir des énoncés les plus simples, dans lesquels figure un seul lexème appartenant à une classe ouverte. Ceci permet de déterminer une ou plusieurs classes distributionnelles de lexèmes. S'il y en a plusieurs, on retiendra tout particulièrement celle qui permet de faire des énoncés assertifs décrivant une action ('agir', 'courir', 'manger', etc.). Les formes qui permettent de faire des prédications, c'est-à-dire de construire un énoncé assertif et de véhiculer la force illocutoire, sont appelées des prédicatifs. Les prédicatifs du français sont essentiellement les formes verbales finies et donc la principale classe de lexèmes fournissant des prédicatifs est la catégorie des verbes.

Une fois établies les catégories de prédicatifs, on définit les substantifs comme les éléments qui peuvent servir d'actants d'un prédicatif, c'est-à-dire les syntagmes désignant les principaux participants d'une prédication, notamment dans une construction transitive où quelqu'un agit sur quelqu'un ou quelque chose. Nous avons vu à la section précédente, que ceci permettait en français de déterminer trois parties du discours en français : les substantifs purs incluant pronoms et noms propres, les noms et les déterminants.

En fait, il n'en va pas ainsi pour toutes les langues. Le cas des langues dites omniprédicatives, que l'on peut illustrer par le nahuatl classique, la langue des aztèques parlée au Mexique au moment de la conquête espagnole, mérite d'être présenté. En nahuatl, on distingue assez facilement verbes et noms, puisqu'il existe une classe de lexèmes qui varient en temps (et désignent notamment des actions) et une classe de lexèmes qui varient en nombre (et désigne notamment des objets du monde). Néanmoins, 
formes verbales et formes nominales possèdent des distributions syntaxiques similaires : les deux fonctionnent comme prédicatifs et les deux fonctionnent comme substantifs lorsqu'ils sont précédés du marqueur in (Launey 1985).

(7) a. $n i-c h o ̄ c a$, lit S1sg-pleurer, 'je pleure'

b. $n i-t i ̄ c i t l$, lit. S1sg-médecin, 'je suis médecin'

c. chōca in tīcitl, lit. pleurer DEF médecin, 'le médecin pleure'

d. tīcitl in chōca, lit. médecin DEF pleurer, 'celui qui pleure est médecin'

Le fait qu'il ne soit pas toujours possible de distinguer noms et verbes uniquement sur la prédicativité amène à faire appel à des critères notionnels pour décider parmi les classes distributionnelles identifiées celles que l'on souhaite considérer comme les verbes et les noms ${ }^{10}$. Croft $(2000: 88)$ propose les définitions suivantes : verb $=$ predication of an action ; noun $=$ reference to an object $;$ adjective $=$ modification by a property. Soulignons que à l'instar du français, le nom ne permet pas toujours à lui seul la référence à un objet et que cette référence à un objet peut-être due à un "déterminant » qui peut en plus, à l'instar du nahuatl, se combiner aussi à des formes verbales pour référer à un objet. Il faut donc prendre la précaution de définir les noms non comme référant à des objets mais comme dénotant des classes d'objets; et définir les verbes comme dénotant des classes d'action, la prédication pouvant souvent être le fait de marqueurs qui peuvent aussi se combiner avec des noms (comme la copule en français).

Si en nahuatl, la prédicativité suffit à elle seule à épuiser toutes les classes ouvertes de lexèmes ${ }^{11}$, en français, prédicatif et substantif n'y suffisent pas. En effet si l'on regarde les éléments dépendant prototypiquement d'un nom, on voit apparaître une nouvelle catégorie, celle de l'adjectif ${ }^{12}$. Et si l'on itère encore une fois le processus et que l'on regarde les éléments dépendant prototypiquement d'un adjectif, on voit apparaître une nouvelle catégorie encore, que nous appellerons pour l'instant des adadjectifs. A partir de là le processus boucle et on n'obtient plus de nouveaux lexèmes : tous les lexèmes qui peuvent dépendre d'un adadjectif (vraiment très triste) sont eux-mêmes des adadjectifs (vraiment triste). Les adadjectifs possèdent une autre particularité en français qui est de pouvoir modifier les verbes et ils forment donc ainsi une sous-classe d'adverbes.

De la même façon que nous avons distingué nom et substantif, il nous semble important de distinguer verbe et prédicatif. Nom et verbe désignent des parties du discours, c'est-à-dire des classes distributionnelles majeures de lexèmes. Ces lexèmes doivent en français être combinés avec des morphèmes flexionnels ${ }^{13}$ pour former des mots. Substantifs et prédicatifs désignent des classes distributionnelles de syntagmes.

Nous avons jusque là contourné la question des adverbes. L'adverbe est le parent pauvre des caractérisations des parties du discours. On a souvent dit que la catégorie des adverbes est une sorte de catégorie poubelle dans laquelle se retrouvent tous les éléments qui n'ont pas pu être caractérisés autrement. Ainsi Riegel et al. (1994:375) : «Les adverbes forment une catégorie résiduelle où l'on range traditionnellement les termes invariables qui ne sont ni des prépositions, ni des conjonctions, ni des interjections. » Nous retiendrons plutôt la définition qu'en donne Schachter $(1985: 20):$ les adverbes sont les modifieurs de verbes, adjectifs et adverbes ou, plus généralement, les modifieurs de tout ce qui n'est pas le nom. Cette classe est assez hétérogène, puisqu'un modifieur d'une des trois catégories - verbe, adjectif ou adverbe - peut ne pas modifier les autres catégories (comme très qui ne modifie pas les verbes, beaucoup qui ne modifie pas les adjectifs ou facilement qui ne modifie pas les adverbes). Il n'y a en fait aucune propriété distributionnelle qui soit partagé par tous les adverbes et il y a même, comme le remarque Creissels (1995 : 135) des couples d'adverbes comme ici et très qui n'ont aucun environnement syntaxique commun. Il ajoute : «il faut accepter de reconnaître que l'inventaire traditionnel des «adverbes » n'est rien d'autre qu'un fourre-tout où on se débarrasse de toutes les unités qu'on est incapable ranger dans une classe grammaticale positivement définie, c'est-à-dire dont les membres ont en commun au moins certains comportements syntaxiques permettant de les caractériser.». 
Dans la suite, nous restreindrons notre emploi du terme adverbe pour désigner la seule classe des modifieurs du verbe. Ceci ne résout pas tous les problèmes. Une telle définition présuppose que les modifieurs du verbe sont distincts des substantifs, qui en occupent les positions actancielles. Or on sait que certains éléments peuvent occuper les deux types de positions, à commencer par les compléments prépositionnels qui peuvent être actanciels ou circonstanciels selon les cas, notamment les compléments locatifs : aller à la plage, poser quelque chose sur la table, dormir dans la rue. Nous allons explorer ce point, mais précisons d'abord quelque chose concernant les prépositions.

Nous avons fait jusque-là comme si chaque position syntaxique était occupée par une partie du discours précise. En fait, des éléments de différentes catégories peuvent venir occuper une position, mais seule une partie d'entre eux peuvent l'occuper sans marquage particulier. Pour celles qui doivent recevoir un marquage, on dit que le marqueur effectue une translation d'une catégorie dans une autre. Par exemple, un verbe peut occuper une position actancielle où est attendu un substantif : Pierre veut [ une pomme + manger une pomme + que Marie lui donne une pomme ]. Pour cela, le verbe doit être à l'infinitif ou former une proposition introduite par une conjonction de subordination comme que. L'infinitif est donc un translatif de verbe en substantif, tandis que que est un translatif de prédicatif en substantif, c'est-à-dire un translatif de verbe fini en substantif. La forme verbale infinitive est ainsi un mot de catégorie biface, verbe pour ses dépendants et substantif pour son gouverneur (Tesnière 1959:418).

Les prépositions sont présentées par Tesnière comme des translatifs de substantifs ou de noms ${ }^{14}$ en adjectif ou en adverbe, car les groupes prépositionnels peuvent aussi bien dépendre d'un nom que d'un verbe. Or les prépositions sont également des marqueurs fonctionnels de substantifs et accompagnent les substantifs dans diverses positions actancielles : parler à quelqu'un, parler de quelque chose, compter sur quelqu'un. Dans ce cas, doit-on considérer qu'il s'agit d'un groupe substantival marqué ${ }^{15}$ (ce qu'on ferait pour une langue à cas) ou de groupe adverbial en position actancielle ? C'est un cas en quelque sorte inverse de celui-là que nous allons étudier : celui bien connu, mais non moins épineux, des substantifs qui peuvent occuper des positions circonstancielles sans marquage.

\section{Noms temporels et adverbes temporels}

Après avoir étudié dans la section 2 les relations entre la notion de substantif et celles de nom, de pronom et de déterminant, nous allons nous intéresser à la relation entre substantif et adverbe. Comme nous venons de le rappeler à la section précédente, substantifs et adverbes se caractérisent tous les deux comme étant des dépendants du verbe, les substantifs occupant les positions actancielles et les adverbes les positions circonstancielles. Comme on sait qu'il existe entre actant et circonstant un certain continuum et qu'il y a beaucoup de compléments du verbe dont le statut n'est pas aisé à trancher, on peut s'attendre à ce qu'il y ait une certaine proximité entre les catégories du substantif et de l'adverbe et c'est effectivement ce que nous allons montrer ici, en nous penchant sur le cas des circonstants temporels. Les lexèmes tel que demain, longtemps ou souvent sont classés par la grammaire traditionnelle dans la catégorie des adverbes. Sans remettre en question leur catégorisation comme adverbe, au sens de modifieur verbal, nous allons regarder plus attentivement leurs propriétés distributionnelles et voir qu'ils ont aussi des propriétés qui les rapprochent des substantifs. Chacun de ces adverbes commute avec des syntagmes nominaux. Plus précisément, on peut construire trois paradigmes de constituants qui expriment respectivement des compléments temporels de date, de durée et de fréquence ${ }^{16}$ et dans lesquels on trouve à la fois des adverbes (cas b) et des groupes substantivaux (cas a) :

(8) date

a. Pierre est venu $[$ ce matin + la semaine dernière + un autre jour + un lundi + la

dernière fois + cet été + l'année dernière $]$.

b. Pierre est venu $[$ hier $+($ très $)$ tard + lundi + alors + dernièrement $+($ très $)$ récemment

+ jadis + autrefois + quand? ]. 
(9) durée

a. Pierre est venu $[$ deux heures + la nuit entière + quelques minutes + un long moment

+ un certain temps + le temps de manger + combien de temps ? ].

b. Pierre est venu [ quelque temps ${ }^{17}+$ (très) longtemps + indéfiniment $]$.

(10) fréquence

a. Pierre venait [ tous les jours + un semaine sur deux + le lundi + deux fois (par

semaine) + chaque semaine $]$.

b. Pierre venait $[$ (très) souvent + quelquefois + parfois + jamais + plus + toujours + encore + (très) fréquemment ].

Ces constituants qui, tout en commutant avec des adverbes et en jouant un rôle circonstanciel, ont la structure interne de constituants substantifs seront appelés des substantifs temporels. De même, nous appellerons les noms temps, fois, moment, jour, semaine, qui combinés à des déterminants peuvent former des substantifs temporels, des noms temporels. Les adverbes demain, longtemps, souvent, parfois fonctionnent plus ou moins comme des pronoms pour cette classe de noms : certains possèdent un emploi déictique comme hier ou demain. Les noms/substantifs de jour comme lundi peuvent être comparés à des noms/substantifs comme maman : lundi peut être utilisé comme nom temporel (le lundi de Pâques à comparer avec la maman de Zoé) ou nu comme déictique indirect (lundi désigne le lundi précédant ou suivant le moment de l'énonciation de même que maman désigne la maman d'un des interlocuteurs) ${ }^{18}$. Si tous n'ont pas un emploi pronominal à proprement parler (c'est-à-dire qu'ils ne sont ni déictiques, ni anaphoriques), ils possèdent néanmoins des valeurs comparables aux éléments que l'on appelle traditionnellement des pronoms indéfinis : par exemple, face à jamais, parfois, souvent, toujours, on trouve aucun, certains, la plupart, tous.

Parmi les adverbes temporels, il y en a qui sont plus « adverbiaux » et peuvent notamment être modifiés par très (voir (8b-9b-10b)). Pour les autres, il a déjà été noté leur proximité avec les noms, par exemple par Creissels (1995) ${ }^{19}$. Voici aussi ce qu'en dit Pinchon (1969) : «Ce qui fait que des mots comme hier, aujourd'hui, demain ne peuvent être rangés dans les substantifs [nos noms], leur caractère invariable, leur impossibilité d'être précédés d'un déterminant spécifique et d'être qualifiés par un adjectif. » L'erreur est ici de vouloir les ranger parmi les noms et non parmi les pronoms qui sont souvent invariables (comme ça ou on), ne peuvent être précédés d'un déterminant, ni être qualifiés par un adjectif.

Le fait que les adverbes temporels sont bien en général des substantifs temporels est confirmé par la possibilité de les employer après une préposition, même si on notera que certains sont moins substantivaux que d'autres. On peut ainsi construire des compléments de date en ajoutant une préposition adéquate devant un complément de durée et vice versa :

(11) date $=$ Prép + durée

Pierre viendra dans $[$ deux semaines + quelque temps + (très) longtemps $]$.

(12) durée $=$ Prép + date

Pierre est ici depuis [ la semaine dernière + hier + lundi $\left.+{ }^{*} t o ̂ t ~\right]$.

Ils peuvent également être utilisés comme complément de nom :

(13) a. date : la réunion de [ la semaine prochaine + demain + lundi $+*$ tard $]$

b. durée : un ami de [ 30 ans + de toujours ].

En même temps, le caractère adverbial des substantifs temporels est indéniable. Ils fonctionnent comme modifieurs du verbe et commutent avec des groupes prépositionnels. Il y a d'ailleurs d'autres noms ou substantifs à valeurs temporels qui doivent obligatoirement être utilisés avec une préposition : 
(14) date : Pierre viendra $\left[\grave{\boldsymbol{a}} 6\right.$ heures moins $10+\grave{\boldsymbol{a}}$ midi $+\grave{\boldsymbol{a}}$ un autre moment + en janvier ${ }^{20}$

+en été + au printemps $\left.{ }^{21}\right]$.

Les substantifs temporels peuvent être sans difficultés coordonnés avec les groupes prépositionnels de même fonction ${ }^{22}$ :

(15) a. Pierre doit retourner chez le dentiste la semaine prochaine et en janvier.

b. Pierre ne sait pas s'il arrivera le matin ou à midi.

Enfin, les adverbes temporels peuvent modifier un adjectif :

(16) des exigences [ parfois + toujours + désormais + aujourd'hui $]$ contradictoires

ce que les noms temporels peuvent aussi, bien que plus difficilement :

(17) a. des exigences chaque jour plus contradictoires

b. des exigences ce matin encore contradictoires.

$\mathrm{Si}$ nous revenons aux noms temporels, on voit qu'ils ont cette capacité à construire des groupes substantivaux modifieurs de verbe. On peut donc considérer qu'il s'agit d'éléments bifaces qui se comportent comme des noms ordinaires vis-à-vis de leurs dépendants (ils prennent un déterminant, sont modifiés par des adjectifs, etc.) et comme des adverbes vis-à-vis de leur gouverneur ${ }^{23}$. D'autres noms (moment, été) ou substantifs (midi, janvier), également à valeur temporelle, demandent une préposition et sont donc légèrement moins " adverbiaux » que les noms et substantifs temporels qui fonctionnent nus. Terminons cette section en notant que si les adverbes temporels sont les adverbes du français qui sont les plus clairement des substantifs, ils ne sont pas les seuls adverbes à avoir des propriétés substantivales. Les adverbes locatifs, par exemple, ne commutent pas avec des groupes substantivaux en position canonique, mais peuvent s'employer après des prépositions, où ils commutent alors avec des groupes substantivaux ${ }^{24}$ :

(18) a. Pierre va [ici + là-bas + où ? + ailleurs + à Paris + en Auvergne + dans le Midi $]$

b. les habitants de [ici + là-bas + où ? + ailleurs + Paris + l'Auvergne + le midi $]$

c. Pierre va vers $[i c i+$ là-bas + où $?+$ ailleurs + Paris + l'Auvergne + le centre-ville $]$

Une autre catégorie d'adverbes possède une propriété assez remarquable qui les rapproche des substantifs : les adverbes de quantité. Ceux-ci peuvent s'utiliser comme déterminants en combinaison avec la préposition de :

(19) $[$ trop + plus + beaucoup $]$ de gens

Et surtout, ils peuvent comme d'autres déterminants avoir un emploi pro-nominal :

(20) $[$ beaucoup + trop + ?plus que prévu + deux + plusieurs $]$ sont venus.

\section{Conclusion}

A travers cette étude centrée sur les adverbes temporels, nous souhaitions montrer que la question des parties du discours, même pour une langue bien étudiée comme le français, est encore ouverte à discussion. Derrière l'étude d'une classe distributionnelle particulière de lexèmes du français, nous nous sommes interrogé sur la stratégie à adopter pour définir les parties du discours est sur les conséquences qu'il faut en tirer pour les parties du discours du français. En rapprochant les adverbes temporels des noms temporels, nous avons vu qu'il y avait un certain continuum entre des adverbes temporels qui sont clairement des substantifs temporels (comme hier) et d'autres qui s'en éloignent (comme tard). Une classification complète des adverbes de verbe (ceux qui jouent un rôle de complément circonstanciel comme les adverbes temporels) reste à faire. 
Un des points qui nous semble essentiel est l'introduction de la catégorie du substantif à distinguer de la notion de nom. Considérer une telle catégorie permet plusieurs choses :

- de pouvoir étudier toute l'étendue des éléments qui se situent entre les pronoms purs comme lui ou ça et les noms propres purs comme Paris ou Napoléon ;

- d'appréhender le groupe substantival sans avoir à décider qui du nom ou du déterminant est la tête ;

- et de faire le lien entre adverbes et groupe substantivaux ayant un rôle circonstanciel.

Notre contribution est loin de clore l'étude des adverbes/substantifs et noms temporels. Il y a énormément d'idiosyncrasies dans le comportement de lexèmes comme fois, temps ou moment et chacun d'eux mérite une étude spécifique. Les adverbes/substantifs et noms temporels possèdent une combinatoire assez complexe (hier matin, tard ce soir, vendredi ler janvier 2010, etc.) dont l'étude permettrait de préciser les différentes sous-classes distributionnelles et les constructions associées.

\section{Références bibliographiques}

Almeida M.-E., Maillard M. (1998). Divergences français/portugais dans le métalangage grammatical et recherche de nouvelles convergences européennes. In Colombat B., Savelli M., Métalangage et terminologie linguistique, Leuven : Peeters.

Creissels D. (1995). Eléments de syntaxe générale, Paris : PUF.

Croft W. (2001). Radical construction grammar : syntactic theory in typological perspective, Oxford: Oxford University Press.

Croft W. (2000). Parts of speech as language universals and as language-particular categories. In M. P. Vogel, B. Comrie, Approaches to the typology of word classes, Berlin-New York : Mouton de Gruyter, 65-102.

Haegeman L. (1994). Introduction to Government and Binding Theory, Cambridge : MIT Press.

Hengeveld K., Rijkhoff J., Siewierska A. (2004). Parts-of-speech systems and word order, J. Linguistics, 40, 527570.

Hopper P. J., Thompson S. A. (1984). The discourse basis for lexical categories in Universal Grammar, Language, 60, 4, 703-752.

Jespersen O. (1924). The Philosophy of Grammar, Chicago : Chicago U. Press.

Kahane S. (2007). La distribution des articles du français. In M. Charolles, N. Fournier, C. Fuchs \& F. Lefeuvre (éds.), Parcours de la phrase - Mélanges offerts à Pierre Le Goffic, Ophrys, Paris, 159-174.

Launey M. (1994). Une grammaire omniprédicative : essai sur la morphosyntaxe du nahuatl, Paris : CNRS.

Le Goffic P. (1993). Grammaire de la phrase française, Paris : Hachette.

Lemaréchal A. (1989). Les parties du discours : sémantique et syntaxe, Paris : PUF.

Molinier C. (1990). Les quatre saisons : à propos d'une classe d'adverbes temporels, Langue française, 86, 1, 46-50.

Pinchon J. (1969). Problèmes de classification. Les adverbes de temps, Langue française, 1:1, 74-81.

Riegel M., Pellat J.-C., Rioul R. (1994). Grammaire méthodique du français, Paris : PUF.

Schachter P. (1985). Parts-of-speech systems. In Shopen, Timothy (ed.), Language typology and syntactic description, vol. I : Clause structure. Cambridge : Cambridge University Press.

Tesnière L. (1959). Eléments de syntaxe structurale, Klincksieck, Paris.

Wilson 1. (1842). Outlines of English Grammar, Londres : Rivington. 
${ }^{1}$ Selon un usage courant, nous utilisons le terme partie du discours pour désigner les principales catégories distributionnelles de lexèmes.

${ }^{2}$ C'est le cas aujourd'hui dans la tradition francophone ou anglophone, mais dans les traditions germanophone ou hispanophone les termes Substantiv ou substantivo sont toujours utilisés pour désigner les noms.

${ }^{3}$ Creissels (1995) utilise le terme de nom auto-déterminé pour désigner ce que nous appelons substantif. Le terme est très parlant, mais nous préférons un terme qui ne laisse pas supposer que la notion de substantif est définie à partir de celle de nom, alors que c'est exactement l'inverse.

${ }^{4}$ Plus exactement, ce sont les formes pronominales, comme $t u$ ou toi, qui fonctionnent comme substantifs. Le lexème pronominal doit quant à lui être fléchi en cas pour former un substantif, ainsi qu'en genre et nombre à la $3^{\text {ime }}$ personne : il(s)/lui/eux/elle(s).

${ }^{5}$ Malgré le caractère figé de le sien (pas de commutation possible sur le), la séquence est compositionnelle et a notamment une double valeur pronominale (puisqu'il oblige a construire deux référents, celui d'un objet et de son possesseur), où chacun des deux éléments, le et sien, porte sa valeur pronominale habituelle.

${ }^{6}$ Par exemple, en nahuatl classique, dont nous parlerons plus tard, les pronoms interrogatifs fonctionnent uniquement en position prédicative (Launey 1985) : tlê in ti-cua, être_quoi DEF S2sg-manger, litt. est quoi ce que tu manges, 'tu manges quoi ?', tandis que *ti-cua in tlê. Ceci peut être expliqué par le rôle obligatoirement rhématique d'un pronom interrogatif, rôle qui dans les langues, comme le nahuatl, qui n'ont pas de contraintes catégorielles sur la fonction prédicative, tend à contraindre la fonction prédicative.

${ }^{7}$ Les pronoms possèdent également des emplois génériques : On ne peut pas lui faire confiance. Si tu lui confies quelque chose, tu n'es pas sur de le récupérer.

${ }^{8}$ L'article défini le/la/les appartient à une classe distributionnelle de déterminants particulière, comprenant également le démonstratif ce et les possessifs (mon, ton, son, ...), qui se caractérisent sémantiquement par l'interprétation définie, morphologiquement par un pluriels en $/ \Sigma /$ (les, mes, ces) et syntaxiquement par la cooccurrence possible avec les numéraux (les/mes/ces deux livres) (Kahane 2006). L'article démonstratif ce/cette/ces possède également une proximité forte avec le pronom ça de forme faible $c e$ et les possessifs entrent tous dans un paradigme pronominal : mon-me-moi, ton-te-toi, etc. Si l'on considère le paradigme complet, on a donc, comme pour l'article défini, trois emplois, comme déterminant, pro-nom et pro-substantif.

9 Voici en préambule ce qu'en dit Le Goffic (1993) : «Nature et fonctionnement sont liés, même s'il est méthodologiquement indispensable de (commencer par) les distinguer rigoureusement. Un exemple de difficulté : dans une phrase comme Beaucoup sont partis, il n'est satisfaisant de dire ni que c'est un adverbe qui est sujet, ni que l'adverbe se transforme en nom. On est contraint à une réflexion sur les rapports entre le nom et l'adverbe dans le domaine de la quantification (beaucoup a une origine nominale transparente). On ne peut dire simplement qu'un terme change de catégorie au gré de ses emplois, mais il s'agit de comprendre en quoi et comment une différence de fonctionnement peut retentir sur la « nature » même d'un terme. »

${ }^{10}$ Insistons bien sur le fait que les critères notionnels ne servent pas à caractériser les catégories, mais juste à les nommer, comme le dit fort explicitement Schachter $(1985: 4)$ : "While it is assumed here that the assignment of words to parts-of-speech classes is based on properties that are grammatical rather than semantic, and often languageparticular rather than universal, it is also assumed that the name that is chosen for a particular parts-ofspeech class in a language may appropriately reflect universal semantic considérations ». Voir aussi Lyons (1968: 317-19), auquel renvoie l'auteur.

${ }^{11}$ Il existe une troisième catégorie ouverte de lexèmes en nahuatl que Launey (1985) appelle les locatifs. Ils se distinguent des noms par le fait qu'ils sont invariables et par leur valeur locative intrinsèque (Mexico ni-câ, lit Mexico S1sg-être, 'je suis à Mexico'). Il existe d'autres éléments lexicaux, notamment des modaux qui se placent avant le mot prédicatif, mais ils appartiennent tous à des classes fermées.

${ }^{12}$ Le terme adnom serait certainement plus approprié. Le terme adnoun est utilisé dans la grammaire de l'anglais de Wilson (1842) et il est courant dans la description du coréen. La raison en est que les éléments qui traduisent nos adjectifs se divisent en deux classes totalement distinctes. Les éléments de la première sont des prédicatifs et se distinguent des verbes d'action uniquement par des propriétés aspectuelles marginales; on peut donc considérer qu'il s'agit simplement d'une sous-classe de verbes, des verbes statiques (par opposition aux verbes d'action qui sont dynamiques). L'autre catégorie, celle de l'adnom, comprend les numéraux et d'autres éléments qui s'apparentent parfois dans leur sémantique à nos déterminants, sans en avoir le caractère obligatoire pour former des substantifs. 
${ }^{13}$ Nous n'avons pas discuté de ce que sont les morphèmes flexionnels du nom dans cet article. Le fait que les noms nus ne s'utilisent généralement pas dans des positions actancielles en français permet de considérer que le nom est indissociable d'éléments qui comme l'article expriment la définitude et que cette catégorie est ainsi une catégorie flexionnelle du nom en français. Cf. Kahane 2007.

${ }^{14}$ La plupart des prépositions ne translatent pas seulement des substantifs, mais peuvent aussi avoir comme complément un nom sans déterminant : patin à roues alignées, livre de syntaxe, cocotte en papier, maison sur pilotis, lettre avec accusé de réception, lit pour enfant, nom sans déterminant.

${ }^{15}$ Si certaines prépositions peuvent prendre comme complément une complétive, c'est-à-dire un verbe translaté en substantif par que (après que tu sois venu, pendant que tu étais là, pour que tu viennes, sans que tu saches), les plus grammaticales ne le peuvent pas (*Pierre pense à que tu viennes, *Pierre parle de que tu viennes, *Pierre compte sur que tu viennes). Cette propriété peut être un argument supplémentaire pour considérer que les prépositions qui ne se combinent pas avec une complétive ne sont pas des translatifs de substantifs, mais bien des marqueurs de substantifs. Un dernier argument est la commutation du groupe prépositionnel avec un pronom : Pierre y pense, Pierre en parle, Pierre compte dessus.

16 Pinchon (1969) considère une quatrième classe qui est l'ordre de succession des actions, marqué par avant,auparavant, après, aussitôt. Il s'agit pour nous d'un cas particulier de date, repéré par rapport au moment par d'une action ou d'un fait. Il existe néanmoins des adverbes temporels qui ne répondent à aucune des trois questions caractérisant la date (quand ?), la durée (combien de temps ?) ou la fréquence (combien de fois ?), comme désormais, ensuite, enfin, finalement.

${ }^{17}$ Nous considérons que quelque temps est figé au même titre que quelque chose ou quelque part, dans la mesure où quelque (singulier) n'est plus productif en français contemporain (*quelque cheval).

${ }^{18}$ A noter quand même que lundi peut être modifié : lundi prochain, lundi dernier, lundi en huit, lundi ler janvier. Mais on a aussi maman chérie, belle-maman ou mamie Odette.

${ }^{19}$ Creissels $(1995: 140)$ est très explicite sur la nature pronominal de ces adverbes : « Beaucoup de ces formes ont de manière évidente un degré d'affinité avec les noms qui les met nettement à part des autres "adverbes ". Si nous comparons par exemple les phrases (i) à (iii), qui contiennent chacune un "adverbe », nous pouvons voir que la phrase (i) est la seule dans laquelle l'" adverbe " se prête à des transformations de mise en relief par ailleurs caractéristiques de constituants dont le statut d'arguments nominaux d'un prédicat verbal est indiscutable — ex. (iv) à (viii).

(i) il est venu hier

(ii) il est venu rapidement

(iii) il est déjà venu

(iv) hier, il est venu

(v) hier en tout cas, il est venu

(vi) c'est seulement hier qu'il est venu

(vii) même hier il est venu

(viii) hier aussi il est venu

On peut expliquer ceci en considérant que hier tend à présenter les mêmes propriétés syntaxiques que le syntagme nominal dont il est le substitut. En effet, on peut poser que hier est mis pour le $x$ dernier où $x=$ jour : le français dit la semaine dernière, le mois dernier, l'an dernier et si *le jour dernier ne se dit pas, c'est précisément parce que le lexique français dispose d'une forme (hier) qui exprime de façon synthétique ce que ce syntagme pourrait exprimer de manière analytique. »

20 On notera également les expressions [ début $+m i+$ fin ] janvier qui permettent aux substantifs de mois de former des compléments de date sans préposition.

${ }^{21}$ Comme l'a noté Molinier (1990), au est ici un amalgame de en $+l e$, tandis que en + la et en $+l$ ' donne en. On retrouve la même chose avec la proposition locative en : en France, en Allemagne vs au Chili.

${ }^{22}$ On observe également pour les compléments actanciels de même fonction des alternances entre substantif et groupe prépositionnel : 
978-2-7598-0534-1, Paris, 2010, Institut de Linguistique Française

Syntaxe

DOI $10.1051 / \mathrm{cmlf} / 2010173$

(i) a. Il lui parle vs Il parle à Marie

b. commencer [ un livre vs à lire ]

c. tenter [ une sortie vs de sortir ].

${ }^{23}$ Cela montre en passant que pour de tels groupes substantivaux c'est le nom qui contrôle la distribution en permettant à ces groupes de modifier un verbe quel que soit le déterminant associé au nom. C'est un argument fort pour considérer que c'est bien le nom qui est la tête syntaxique du groupe substantival ou, en tout cas, que ce n'est pas le déterminant qui en est la seule tête syntaxique.

${ }^{24}$ Les noms utilisés dans les adresses peuvent être utilisés nus pour former des compléments locatifs, mais prennent un déterminant après une préposition :

(i) a. Pierre va [ rue de Lille + place Clichy ]

b. les habitants de [ la rue de Lille + la place Clichy]

c. Pierre va vers [ la rue de Lille + la place Clichy].

Il s'agit d'un comportement différent de celui des noms temporels, mais pas moins remarquable. 